\section{Tissue Microarrays}

The search for biomarkers indicative of disease state or its progression requires large studies for statistical power. DNA arrays are well suited in scale, but suffer from the disadvantage of measuring a surrogate of protein level. Moreover, archival, fixed tissue samples do not make good sources of cDNA for traditional microarrays. By contrast, tissue microarrays can take full advantage of the vast amount of archived clinical material already available. In an authoritative review, Simon et al. (p. 98) describe the enormous promise of tissue microarrays for applications from drug development to patient prognosis. The authors trace the development of the technology, discuss the issue of tissue representativity, and describe the many applications of the technique. The authors conclude with an analysis of the prospects for automated analysis of tissue microarrays. The result is a comprehensive treatment of a technique that can be expected to be a powerful tool for translational research.

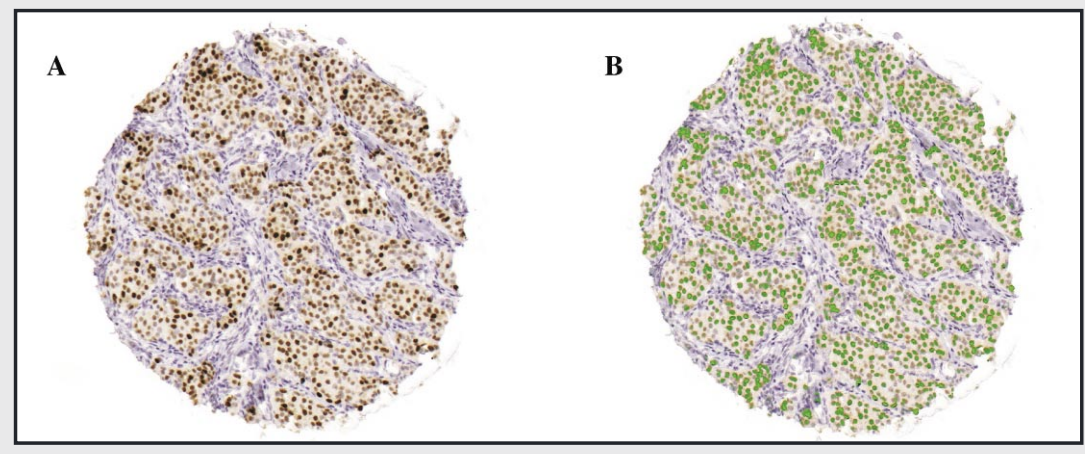

\section{Selling SELDI}

Peptide identification and analysis are important elements of proteomics research. Precursor peptide hormones often differ at the carboxyl terminus to allow for altered enzymatic regulation and cleavage to the active hormone moiety. Methods describing the use of carboxypeptidase $\mathrm{Y}$ (CPY) to digest prohormone and matrix-assisted laser desorption/ionization time-offlight (MALDI-TOF) mass spectrometry to analyze the resultant peptide sequences have been described, but peptide identification is often difficult in complex samples containing adjacent peptide ion peaks. Cool and Hardiman (p. 32) extend carboxyl-terminal sequencing analysis to surface-enhanced laser desorption/ionization time-of-flight (SELDI-TOF) mass spectrometry, which allows for its application to complex mixtures of peptides and proteins (tissue homogenates). The authors provide an additional tool for the investigation of prohormone processing in order to understand the regulation of endocrine protein hormone pathways.

\section{Double Disruption}

Gene targeting is a powerful genetic tool that has been widely used to dissect gene function in diverse organisms. Efficient implementation of this approach can be plagued however by the requirement for multiple sequential knock-outs of single genes and by the paucity of selectable markers in some organisms, such as Dictyostelium. Betapudi et al. (p. 106) describe an approach whereby Dictyostelium discoideum cells are transfected with a mixture of plasmid vectors containing cassettes targeting two different genes (here myosin heavy chain kinases $A$ and $B$ ) followed by PCR-based screening for only one selectable marker (blasticidin-resistance.) The authors find that $2.5 \%$ of all colonies positive for the single marker contain knock-outs of both targeted genes. This gene targeting strategy coupled with rapid PCR-based screening will facilitate the creation of multiple gene knockouts in Dictyostelium and other organisms where the availability of selectable markers is limited.

\section{Helper Probes Chip In}

Annealing of a "helper" oligonucleotide probe directly adjacent to a labeled detection probe can enhance hybridization specificity and signal intensity in various nucleic acid detection schemes. Barken et al. (p. 124) were therefore curious what effect helper probes might have on a bead-based sandwich hybridization assay. In this system, a target mRNA is captured by one probe immobilized on a magnetic bead and detected by means of another probe that is labeled with digoxigenin (DIG). RNA molecules, which bind both probes, are quantified by using an electrical chip sensitive to a reaction product catalyzed by an anti-DIG alkaline phosphatase conjugate.
The researchers systematically examined the effects of helper probes targeted to the regions just adjacent to the capture and detection probes. They found that the helper probes next to the capture probe had more of an effect than the detection probe-associated helpers, but that the contributions of all four helpers were additive. The authors show an impressive 10- to 30-fold enhancement of hybridization when the four helper probes were added to reactions in which rRNA or mRNA targets were detected in the context of total bacterial RNA. This work should further improve detection limits of hybridization-based biosensors.

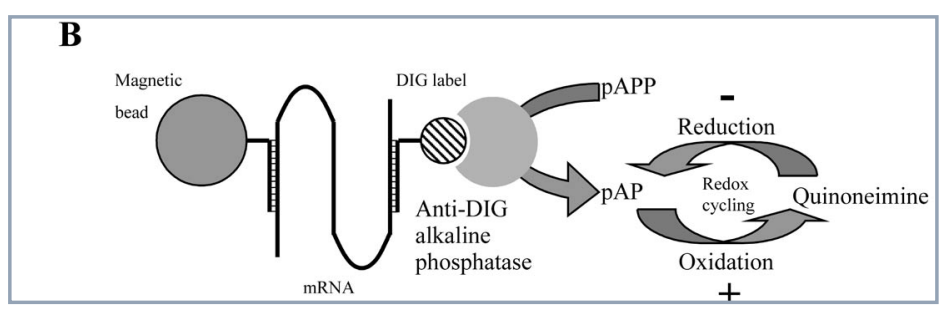


In This Issue

\section{MACSimizing Baculovirus Through-Put}

Baculovirus-based recombinant protein expression systems have become increasingly convenient over the years. Even so, efficient protein production is hampered by slow, work-intensive steps, such as selection of baculovirus-infected cells and determination of virus titer. Mindful of these drawbacks, Philipps et al. (p. 80) describe a new baculovirus expression vector that allows magnetic separation of infected cells and titer determination by green fluorescent protein (GFP) visualization. The new vector contains a CD4-enhanced GFP (EGFP) fusion protein driven by a late viral promoter. Infected cells will express CD4, which allows magnetic cell sorting (MACS) by using magnetic beads coated with anti-CD4 antibody. This simple step results in a 20 -fold enrichment of infected cells, thus generating a higher titer baculovirus stock. In addition, titer determinations themselves are more straightforward, since plaque assays are not required and infected cells can be easily scored by GFP fluorescence. Importantly, the authors show that the expression of the CD4-EGFP fusion protein does not lessen the expression or activity of a test protein. All told, the new baculovirus vector shaves off at least 6 days from a typical procedure for the preparation of virus stock, making protein production in insect cells even more attractive.

\section{Labeling Probes}

High-quality fluorescence-labeled cDNA and oligonucleotide probes are critical for obtaining accurate reproducible data when employing techniques that rely on nucleic acid hybridization, such as microarray analysis. Traditional approaches for direct incorporation of labeled nucleotides into DNA typically result in the incorporation of two to four dye molecules per 100 bases, and incorporation rates can vary among dyes. Cox and Singer (p. 114) have optimized an alternate approach to dye incorporation-a two-step method that involves incorporation of a nucleotide analog that contains a primary amine group, followed by chemical labeling with an aminereactive fluorescent dye. Their data include a demonstration that probes containing eight dye molecules per 100 bases generate the brightest signals on dot blots and human chromosome spreads, independent of the nature of the individual dye. The findings reported here will contribute to the ongoing evolution of hybridization technology.

\section{HLA Hoopla}

Mutations in human leukocyte antigen (HLA) alleles are linked to a variety of disorders, including inflammatory bowel disease, multiple sclerosis, and diabetes. Current HLA genotyping methods include groupspecific PCR, genomic or CDNA sequencing, and hybridization methods, yet none have been successfully scaled-up for use in routine patient diagnosis. In addition, standard single nucleotide polymorphism (SNP) genotyping methods have significant problems with the highly polymorphic HLA sequences and are therefore not suitable for HLA genotyping. Hampe et al. (p. 148) developed a high-throughput HLA genotyping method that combines allele-specific PCR with nonelectrophoretic PCR product detection. The authors successfully validated their method by genotyping the DRB1-HLA locus (which contains a high density of SNPs and is especially difficult to analyze using standard genotyping methods). This high-throughput method allows genotyping of thousands of patients at a reasonable cost and can be automated and integrated into an electronic information system.

\section{Transcription Rates Run Ahead}

Metabolic labeling of nuclear RNA in a run-on assay is commonly used to assess RNA transcription rates. Because this assay requires isolated or cultured cells, analyzing transcription rates in situ has been very difficult. Cui and Tseng ( $p$. 134) found that the quantity of $47 \mathrm{~S}$ pre-rRNA correlates positively with the rate of rRNA transcription and can serve as an indicator of rRNA transcription in commonly used cell lines. The authors further applied this method in an in situ hybridization scheme and demonstrated its validity in vivo in mouse ovary and retina. A potential application of this method is the monitoring of the rate of mRNA transcription in single cells within a tissue of complex cellular composition.

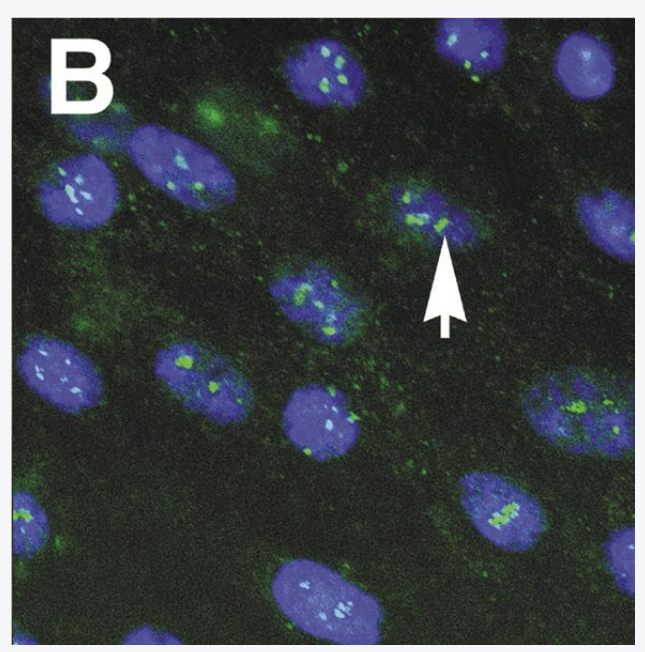

